ISSN 2693-2490

\title{
Neural Experience of Conscious Time
}

\section{Journal of Psychology and Neuroscience}

\section{Gerard Marx*, Chaim Gilon}

MX Biotech Ltd., Jerusalem, Israel.

Institute of Chemistry, Hebrew University, Jerusalem, Israel.

\section{*Correspondence author}

\section{Gerard Marx}

MX Biotech Ltd

Jerusalem

Israel.

Submitted : 21 Aug 2021 ; Published : 3 Sep 2021

\begin{abstract}
"Pure perception and pure memory constantly intermingle"
\end{abstract}

Henri Bergson, 1908.

One can consider that "Time" and "memory" are related experiential facets of mentality. Without memory, there is no Time. To clarify, we distinguish between the physisist's objective time (pTime), which has no emotive quality or memory component, and the subjective conscious time (cTime), which engages both emotions and memory.

Our tripartite mechanism of a neural memory involves neurons interacting with their surrounding extracellular matrix (nECM). Incoming perceptions are chemically encoded in the nECM as metal-centered cognitive units of information (cuinfo), wherein NTs serve as molecular encoders of emotive states

In the context of the tripartite mechanism (Marx \& Gilon, 2012-2020), we consider two possible modes whereby the temporal sequence of events (i.e. cTime) could be recalled by the sensing neural net.

1. Chemical (allosteric) sensing of cuinfo in the nECM by neural receptors (i.e. GPCR, integrins, etc.) which establish fleeting contact with the nECM as they diffuse along the neural membrane. Effectively, this is a lateral decoding process.

2. Electrodynamic sensing of cuinfo vertically displaced from the neural surface. New nECM components and cuinfo are constantly being formed, like coral growths, extending from the neural surface. The individual neuron senses and decodes the distal cuinfo in the surrounding nECM (like long-distance radar detection). Neural sensing is consolidated and transformed by the net into comprehensive memory.

These speculations suggest experimental tests to measure the interactions of the tripartite components, to examine the electro-chemical aspects of neural encoding of memory perceived as cTime.

Keywords : Time, Sensing, Chemodynamic, Electrodynamic, Emotive Memory, Neural Code.

\section{Background}

Many have puzzled over the enigmas of memory and Time.

I thirst to know the power and the nature of time.

Augustine (Christian theologean, philosopher, 354-430 AD)

Smart, 1979

What then will memory be? Nothing but a sensation of impressions in the brain.

Spinoza (Jewish philosopher, 1632-1677) Lin 2005

Time comes to be apprehended as a Quantity, and so falls under the consideration of Geometry and Mensuration.

Robert Hooke ( $1^{\text {st }}$ scientist 1682)

Singer, 1976

We arrive at the idea of Time by connecting what is contained in the province of memory with what is sense-perception.

Ernst Mach (Physicist and philosopher, 1900)

Smart, 1979

Space by itself and Time by itself, are doomed to fade away into mere shadows, and only a kind of union of the two (as SpaceTime) will preserve an independent reality.

Hermann Minkowski (Mathematician, teacher of Einstein, 1908)

Smart, 1979

Time is an idol of language.

Henri Bergson (Philosopher 1908).

Bergson, 1908 
We recognize that the word "time", has multiple definitions, depending on its usage.

For example, the circadian (24 hr) clock in biology, has a fundamental role in regulating the daily physiology and instinctive drives of all animals and plants (Wray, 2015; Ray et al, 2020). Solar and lunar oscillatory processes underlie our unconscious behavior, but do not reflect how we consciously remember the timed order of events.

Perception provides an intuitive approach to comprehend the process called "Time", yet falls short of grasping its actuality. Philosophers, physicists and mathematicians (i.e. Hooke, 1682 in Singer, Augustine (in Smart, 1979), Bergson, 1908; Spinoza (Lin 2005), Schrodinger (Schrodinger, 1944; Rosenblum \& Kuttner, 2011; Brann, 2017); Husserl (in de Warren, 2016), Descartes (in Joyce, 1971) and von Neumann (Taub, 1950; Sherry \& Schacter, 1987; Tulving, 2002; Tononi, 2008) to name only a few, have grappled with the enigma of Time. But we are still at a loss to mechanistically describe the objective as well as the subjective qualities of Time (1-19). (Fischer, 1967; Brown, 1990; Singer, 1994, 1995; Tononi, 2004, 2008; Bueti, 2011; Howard, 2012; Pockett, 2014; Maniadakis \& Trahanias, 2016; Street, 2016; Buzsaki \& Llinas, 2017; Burdick, 2017; Gupta \& Merchant, 2017).

\section{Q: For neural consciousness, is there absolute Time or is it} all "relative"?

In the computer chip, "information" (info) is embodied materially as dots, dashes, holes, spins, quantal states or as dopants distributed in a memory material (Di Ventra \& Pershin, 2011). Each bit has an energy cost equivalent to the loss of heat upon erasing. Each is meaningless by itself and only achieves meaning when presented as part of a recognized data set, termed "information" (Tononi, 2008). It has been shown that information (info) is ultimately physical (Landauer $1991,1996)$. And associated with an energy cost $(\mathrm{E}=\mathrm{kT} \ln 2$ joule/bit) (Shannon, 1948). The bit has no emotive signifier or meaning; each bit is like the other; none has inherent value/ significance.

By analogy, we have proposed that "cognitive information" (cog-info) leading to neural memory also has a physical aspect (see the cuinfo of the tripartite mechanism, Marx \& Gilon, 2012-2020). But "Time" is incorporal, without the "baggage" of mass or space; neither is it a signal.

\section{Time Information}

For "information" to be recognized as such, it requires an observer or detector.

One could argue that the "observer" of information is not necessarily a conscious being. In computers, the "detector" is an algorithm that recognizes a particular pattern of bits. Bacteria communicate with one another by exchanging signaling molecules (sic. "information") that they sense with receptors (i.e. GPCR). Effectively, they "observe" (detect) the molecular signal (information) sent by others and act thereon. Interestingly, the signaling molecules employed by bacteria (biogenic amines, amino acids) are also used by the neurons (Table 2). This speaks to the universality of biological signaling of information.

Similarly for psychologists, the big mystery relates to the processes that result in the subjective self, the neural EGO. Here too, uncertainty reigns, though life decisions must be made by the neural creature striving to survive.

Physicists adopted the notion that objective time (pTime) is Relative (see Einstein) but ignored the experiential relativity of neural conscious time (cTime). They propose that pTime began in a Big Bang which expanded in a direction defined by Eddington's "entropic arrow", which never ends (Muller, 2016). They developed a variety of clocks, watches of ever greater precision (Gerstner, 2012). But the timekeepers were mum regarding the emotive qualities of conscious time (cTime (see Table 1)

Table 1: Comparing Time.

\begin{tabular}{|l|l|}
\hline pTime & cTime \\
\hline Objective & subjective \\
\hline Demotive & emotive \\
\hline Instant & memory \\
\hline
\end{tabular}

For example, electronic artifacts lack an affective mode of representing emotive cognitive information. A mathematical equation (Fodor, 1975, 1983; Arber, 1987; Franklin, 1995) or a unary algorithm (Howard, 2012) or a binary algorithm for robots (Hasson, 2011) cannot conjure up a psychic state called 'emotion' experienced by neural creatures. Some have attempted to close the gap between human mentation and electronic data processing by harnessing quantum computers (QCs) (Cho, 2019). Though QCs are faster than standard computers, but there is no quantum state equivalent to an emotive state.

Emotive memory involves recalling a previously achieved psychic state. We have suggested that a neuro-chemical code addresses the issue of "emotive memory". Neurotransmitters (NTs), molecules that are synthesized and selectively secreted by the neurons. They elicit both physiologic reactions (body) and psychic effects (mind) (Table 2). With respect to the latter, NTs are employed by neurons to encode emotive (psychic) qualities of cognitive information to memory.

\section{Dimensions}

Consider that each NT molecule (out of 80) induces a unique set of physiologic changes linked to emotive states. Each NT can be considered to introduce another mental dimension. Unlike the binary computer which represents only 2 dimensions (i.e. 0 1), the neural net has available more than 80 biologically active molecules (Table 2) to construct the dimensions of emotive neural memory. 
Table 2

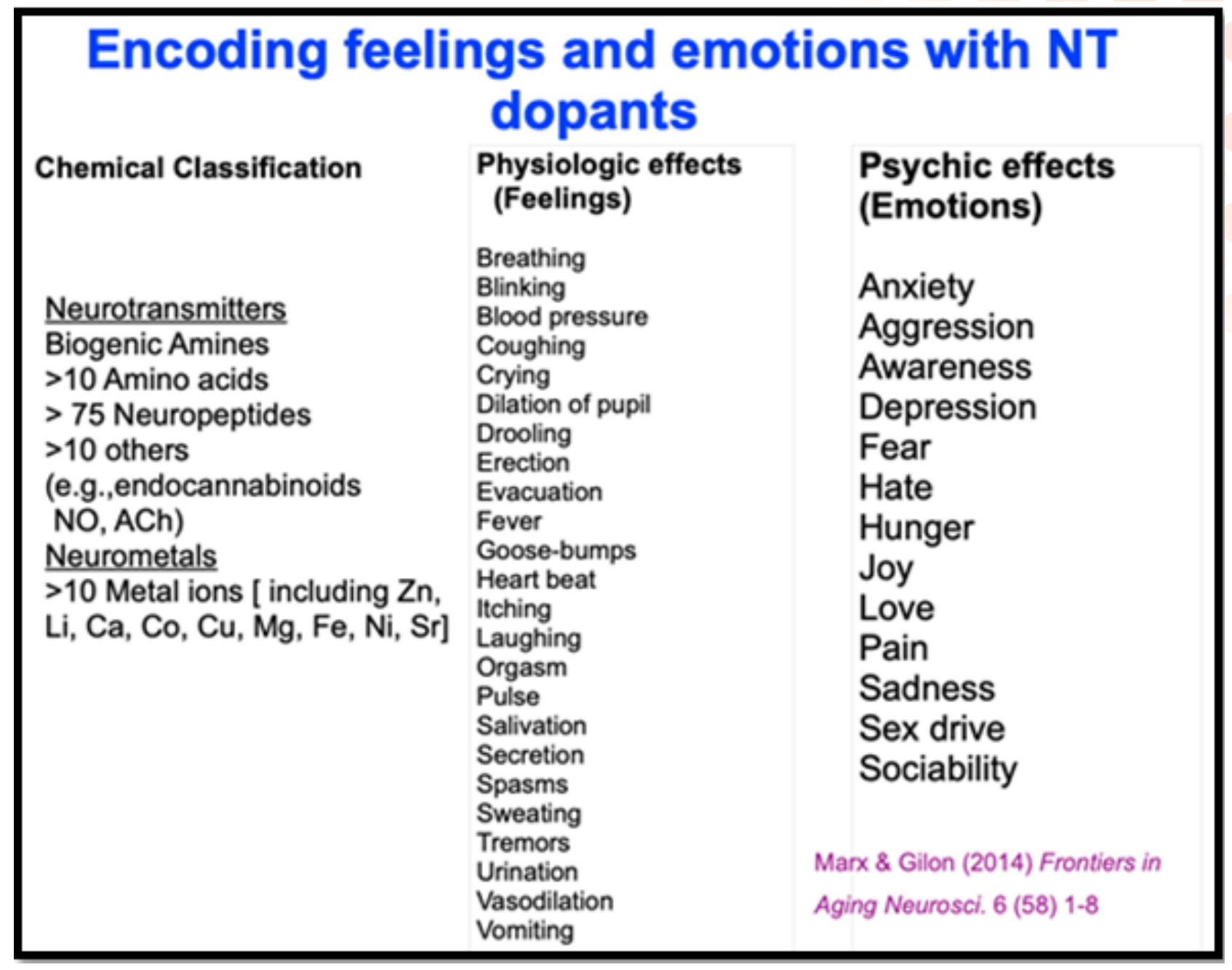

No memory

Require memory

Q: What is the difference between Past and Future?

There are physical traces of the former (i.e. geologic layers, archeologic finds, historical records, photographs, videos, etc.) but none of the latter, except in faith, prayer and mathematical projections. And the Present is fleeting.

Life is all memory, except for the one present moment that goes by you so quickly you hardly catch it going.

Tennessee Williams (Playwright, 1911-1983)

$Q$ : Who is subjective, neural observer that remembers the timing of events?

The nature of consciousness and memory is debated in the pages of neuroscience journals and speculated upon by psychologists and philosophers (Bergson, 1908; Brown, 1990; Block et al, 1997; Kim, 2000). Interestingly, these mirror the debates of quantum physicists and mathematicians over the nature of objective "fact" (Arbib, 1987; Franklin, 1995; Rosenblum \& Kuttner 2011; Muller, 2016). In the reality of quantum physics as well as in everyday experience, one needs an observer to establish a "fact" or to recognize information. Without an observer, there is neither "fact" nor information.
Even with an observer, the physicist is ultimately left only with the "uncertainty" quantified by Heisenberg.

$$
\text { Uncertainty }=\Delta \mathrm{x} \Delta \mathrm{p} \sim \mathrm{h}
$$

where $\mathrm{x}=$ position; $\mathrm{p}=$ momentum calculated as (distance/ time)*mass; $h=$ Planck's constant. For the physicist as well, Time, space and momentum, are inherently uncertain, though linked by Planck's constant.

\section{Time}

Quotations from Augustine resonate:

- $\quad$ Time measures motion, so motion measures Time.

- We measure neither times future, nor times past, nor times present, nor times passing by; and yet we do measure them.

- I measure the motion of a body by Time, but the Time itself I do not measure.

- It is in you, O mind of mine, that I measure the periods of Time.

Interestingly, Augustine related "Time" to "language", ascribing to the New Testament view ("In the beginning was the Word, and the Word was with God, and the Word was God." 
(John 1:1-2). "Beginning" implies a "before" as well as an "after". As only humans employ words, Augustine considered that the onset of Time and God coincided with the human talent for words. Time was a linguistic quirk... a product of language.

By contrast, the Hebrew Bible begins: 'In the beginning, God created the heavens and the earth" (Bereshtit 1:1).

Time related to the material formation of the universe (beginning with light, stars and planets) followed by animal life and the evolution thereof. This is more in keeping with the perspective of modern cosmologists (who measure Time as of the "Big Bang" formation and expansion of the Cosmos some 13.5 billion years ago) and of biologists (who consider animals as an evolving expression of life starting $\sim 4$ billion years ago, starting with bacteria (Coleman et al, 2021)). Birth and death define the limits of lifetimes. Of course, the "Beginning" remains shrouded in the mystery of God, that so captivated Augustine (Smart, 1975) and Spinoza (de Warren, 2016; Lin 2005).

\section{Physics of Time (pTime)}

For the classical physicist, Time was measured by the movements of the sun and moon, the dripping of water, the swing of a pendulum, the unwinding of springs with escapements driving gears, each technique providing ever smaller quantifiable measures of an elapsed period (pTime). But Einstein and Minkowski shattered the concept of absolute Time and Space, neither of which would survive as independent dimensions, but only as merged "Space-Time" (see Minkowski's essay in Smart, 1979). "Relativity" revealed that the gravity fields around suns and planets modulate the fungible aspects of both Space and Time (Space-Time). For Einstein, the speed of light was absolute, but the pTime of observers moving at different speeds relative to one another was not....it contracted or expanded depending on their relative movement. But today, some physicists question Einstein's assumption of the absolute speed of light ("Einstein's Biggest Blunder," on Netflix, D. Sington, dir. 2000).

Quantum physicists have even greater complexities to explain. They must cope with a "no-Time" event in the entangled reactions of physically distant particles which respond to each other's state at "faster-than-the-speed-of-light interactions", which Einstein termed "spooky" (Street, 2016; Cho, 2019). They found that the sub-atomic particles called "electrons" have counterparts called "positrons", considered by Wheeler \& Feynman to be electrons moving "backwards in Time" (Wikipedia).

Some have called upon the quantum theory of evanescent photons to explain memory storage of information in the brain (Buzsáki \& Moser, 2013; Torday \& Miller, 2016). But aside as a metaphorical reference ("a light went on in my mind", Figure 1), this seems farfetched, as there are no visible photons emanating from the brain which is opaque to light nor are they generated in the brain. Of course, all molecular processes are ultimately grounded in the quantum mechanics of atoms and electrons (Pauling, 1960). But this realization does not clarify the neurochemical basis of mental processes.

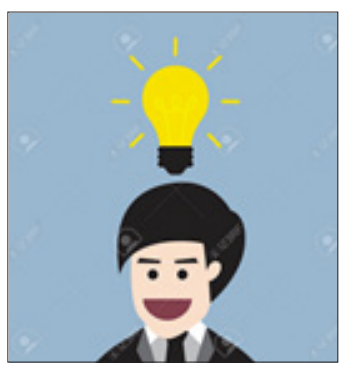

Figure 1: Visual metaphor (lightbulb) for thinking up an idea.

In fact, the brain emits no photons but the consciousness of the observer is not at all uncertain.

Others have considered representing Time in connectionist models, as an effect on the processing of input (Elman 1990). For example, in "feed forward networks employing hidden units and a learning algorithm, the hidden units develop internal representations for the input patterns that recode those patterns in a way which enables the network to produce the correct output for a given input" (Figure 2).

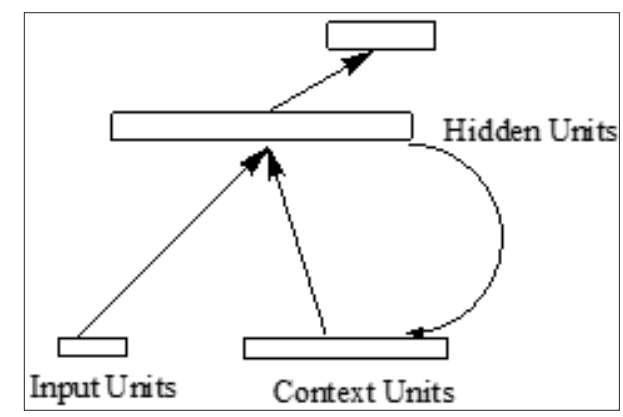

Figure 2: A simple recurrent network in which activations are copied from a "hidden layer" to the context layer, on a one-forone basis, with a fixed weight of 1.0 (adapted from Elman, 27).

Elman states that "the internal representations that develop are sensitive to temporal context; the effect of time is implicit in these internal states." But what are"internal representations"?

\section{Does Figure 2 make physiologic sense?}

The philosopher Hussserl presented a schema for time (Figure 3). This was a brave attempt to concretize the psychic state of temporal passage with geometric logic (Transcendental Phenomenology described by Wittmann, 2016; de Warren, 2016) but it was not explanatory. 


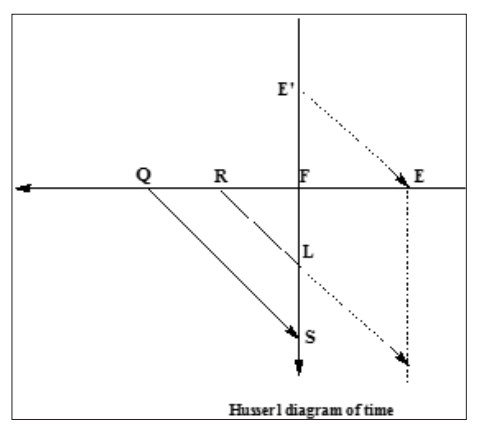

Figure 3: Husserl's “Diagram of Time”. (Adapted from Brann, 1999).

Moreover, the issue of emotions and their connection to Time was totally ignored (more on this later).

For the computer, Time is set by an internal battery clock or an external power source (i.e. $60 \mathrm{~Hz}, 110$ voltage) with reference to Global (UTC) Time. An input is logged in with a date: time stamp that permits the system to assign each input unit with a specific indicator that permits temporal ordering of serial input (Burdick, 2017).

Based on electronic devices capable of Time-informed signal switching, two main approaches have been used to model how the brain represents "Time". One essentially represents duration as pulses that are emitted regularly by a pacemaker that are temporally stored in an accumulator. For example, the Striatal Beat Frequency (SBF) model assumes "time" perception to be the result of the coincidental activation of basal ganglia neurons by cortical neural oscillators. A second approach includes intrinsic explanations, where "Time" is described as a general and inherent property of neural dynamics; Time is intrinsically encoded in the activity of networks of neurons. Both models focus on estimating the duration of events (reviewed by Maniadakis \& Trahanias, 2016). However, both models lack any reference to memory or the emotive aspects of Time.

A co-evolutionary neural network was developed to describe a brain-inspired duration perception system (Maniadakis \& Trahanias 2016). Continuous Time Recurrent Neural Networks (CTRNNs) modules were used to represent knowledge in terms of undefined "internal neurodynamic attractors". Mathematical formulation and graphic representation of such modules (see Figure 4) resulted in complex mathematical functions and flow diagrams whose connection to the physiology of memory and Time remained obscure.

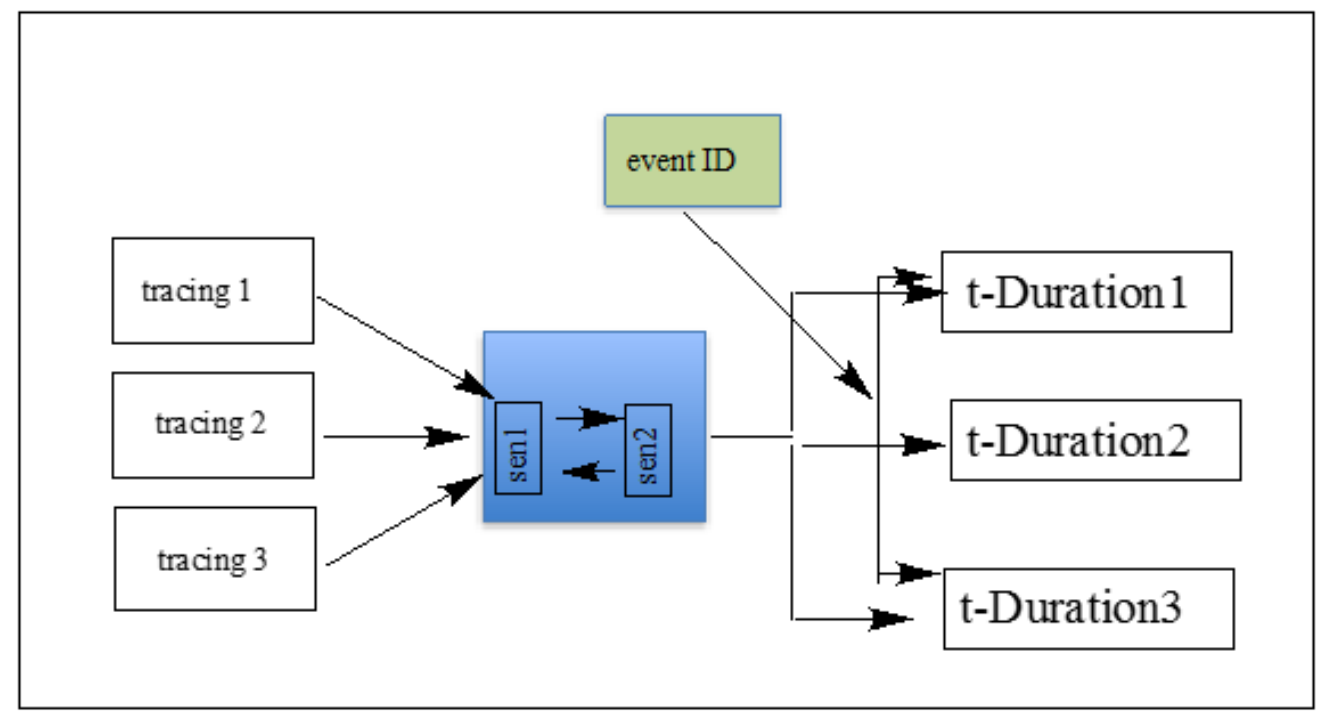

Figure 4: Schematic structure of interval timing (adapted from Maniadakis \& Trahanias, 2016). It is difficult to extract a physiologic sense of cTime from such a representation.

\section{Neuro-biology of conscious time (cTime)}

For the neural creature, cTime is always relative. In that a modern description of any physiologic process necessarily invokes chemistry, so too one must enlist neuro-chemistry to describe temporal perception, particularly in this age of timealtering drugs (Peters et al, 2018). The confluence of psychic states, drugs and neuro-chemical processes cannot be ignored.

Chemists have not been particularly involved in timekeeping or in developing tools to quantitate time, though kineticists measure the rates of reactions (energy of activation) using clocks and thermometers built by physicists are used to measure reaction duration and temperature. There is a class of oscillating (Belousov-Zhabotinsky) reactions that have been described as "clocks", but these are really in-homogeneous mixtures that form visible reaction patterns and were never used to measure Time (Vitz, 2007).

Some have proposed that the recording (imprinting) of memory is a physical change in existing molecules (Katchowlsky \& Neumann, 2012). But the authors were at a loss to present a biologically relevant mechanism. They grasped at "hysteresis" as a possible process, defining hysteresis as:

"A mechanism whereby a dependent variable lags behind changes in an independent variable". 
As examples of such, they pointed to magneto-mechanic circuits and the $\mathrm{pH}$ titrations of macromolecules such as mRNA, polyA + poly U (1:2 ratio). But this is unsatisfactory from a few perspectives. Emotive memory cannot be based on magneto-mechanic effects which are neurologically nonexistent and emotionally barren, nor on local $\mathrm{pH}$ changes, as the system is highly buffered and the local $\mathrm{pH}$ states would be unstable, not capable of persisting as memory (see Amtul \& Rahman, 2016).

\section{"Haiku to Emotions" \\ Emotions are affective, rather than "demotive". Not circumscribed by binary algorithms, But expressed as Passion, Need and Mood. Between 0 and 1, no room for Emotion.}

Symposia have been held on the theme of "Time" (Smart, 1964; Fischer, 1967; Salk Institute Ipsen Symposium, 2018). But glaringly absent were significant discussions of conscious cTime as related to the chemical workings of the brain.

For the neural creature, cTime begins with birth (birthday) and ends with death. The passage of cTime is intertwined with affective states. There is a lifelong succession of "now" experiences, subjectively considered as a "good time" or "hard time" etc.; death being the end of subjective cTime. While alive, our stock of memories of past events (cTime) is continually being augmented by present experience. Memory deteriorates, sometimes forgotten, pathologically in Alzheimer and Parkinson's disorders. Drugs also affect memory, suggesting an underlying chemical basis for neural cTime.

Subjective cTime moves inexorably to the future as one ages, never backward. Melo et al (2015) observed that striatal rat neurons fired at delays spanning tens of seconds and that this pattern reflected the interaction between Time and the animals' ongoing sensorimotor state. Surprisingly, cells rescaled their timed responses when intervals changed, indicating that striatal populations of neurons encoded relative time. But the neural response pattern did not reveal memory's underlying "Time code" nor that of emotions, the visceral states that dilate or contract experiential cTime. There is no time-reversal in the lifetimes of neural creatures, no eternal youth, no going back a la Dorian Gray (a play by Oscar Wilde, 1890).

Is there personal cTime before birth or Time after death (referred to by some as the "hereafter")? Wishful thinking, religious imaginings and playful metaphors do not explain the processes occurring in our heads which order the temporal sequence of our memories.

Equally, logical flow sheets, binary algorithms whatever the pattern (Ellman 1990; Mello 2015), cannot encode emotive events. One must look elsewhere for a satisfactory rationale.

We point to modulating molecules, called neurotransmitters (NTs), that elicit a combination of physiologic reactions and psychic states called "feelings", but which themselves are not molecular clocks. NTs permit one to analytically describe the molecular processes underlying the temporal sequence of recalled subjective states.

The question remains:

How is the temporal order of an emotive event stored for recall?

\section{A Tripartite Mechanism of Neural Memory}

Memory is an inherent aspect of the consciousness of time. We refer the reader to our previous descriptions of the tripartite mechanism of neural memory (Marx \& Gilon 2012-2020), summarized as follows:

\section{Materials}

- Neurons - morphologically spindly, arborized, synaptically and non-synaptically connected signaling cells, all suspended in nECM, some with synaptic contacts between them. Astrocytes and glia which comprise $~ 90 \%$ neural cells, are also involved, but they can be classified as "helper cells", which enable the functioning of the cells comprising the signaling neural net (Davila et al, 2013; Perea et al, 2009; Li et al, 2013).

- nECM - neural extracellular matrix (Schmitt \& Samson;1969; Barros et al, 2011; Kamali- Zare \& Nicholson, 2013), a hydrogel lattice, comprised of copolymers of anionic glycosaminoglycans (GAG) and proteins enswathing all neurons it has been visualized by histologic stains, antibody staining, and electron microscopy and chemically analyzed. The nECM is continuously being added to by biosynthesis.

- Dopants - diffusible metals and neurotransmitters (NTs) released from neural vesicles into the nECM during signaling, which form metal-centered complexes; used by the neurons to encode cog-info. Dopants (metals and NTs) in the brain have been detected and analyzed by HPLCMS, X-ray fluorescence, atomic absorption, neutron activation and immunofluorescence techniques.

The molecular-scale dynamics of the dopant interactions are much faster than the rate of neural processing and firing (Table 4), much like a film with 24 frames per sec, or pixilated, pointilistic color vision, which we perceive as fluid motion or blended color. Each action-NT binding event can count as reading a single unit (cuinfo).

Thus, the components of Neural memory can be divided into two categories:

- Materials (Hardware) - neural net enshrouded in nECM along with dopants (metal cations and neurotransmitters)

- Process - Memory emerges from the dynamic interactions of a neural net with the nECM and dopants (metal cations and NTs).

Some suggest that only integrated information relates to conscious memory (Tononi, 2004, 2008). That is, a single unit of data (a bit) is not really revealing. It needs to be integrated with many others for a meaningful pattern to emerge. This requires an integrative, consolidation process. For example, a 
binary pixel is an objective unit of data that can be transmitted through tele-communications channels, subject to the rules of Turing and the thermodynamics of Shannon and have a material aspect. Physically, the binary bit is encoded as a dopant, hole, pit, spin etc. placed in a matrix (Landauer, 1991; Shannon 1948). The cuinfo (tripartite mechanism) is more complex, but less defined, but also requires a physical embodiment (Street, 2016).

The tripartite mechanism proposes a "chemo-graphic" notation to describe metal-centered complexes of cognitive units of information (cuinfo) (Figure 5). These are the basis of the neural memory code. For the sake of graphic simplicity, we employ a square configuration, but could also have used a pentagon or hexagon, etc.

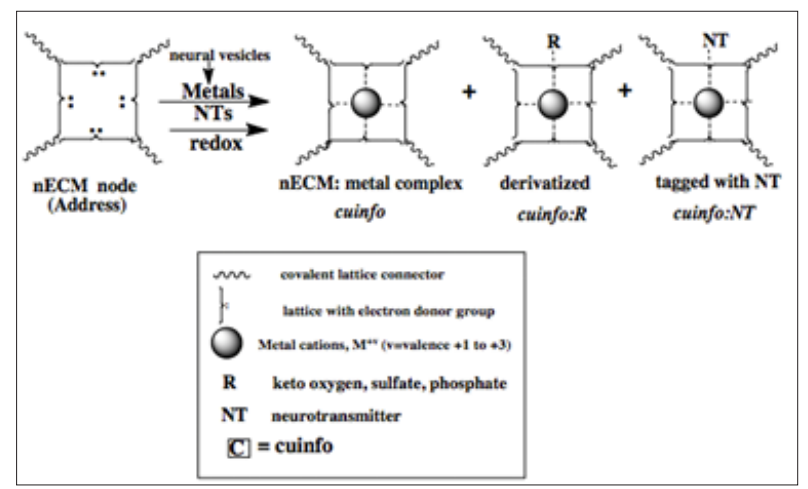

Figure 5: Chemographic representations of the reaction of a nECM (electron rich site) binding site for a metal cation, an "address". The additional binding of an electron-rich neurotransmitter (NT) to cuinfo confers emotive context. The recall of cuinfo by an individual neuron is a chemodynamic process that is communicated electro-dynamically to different anatomic regions.

\section{"Feelings" and "Emotions"}

The terms "feeling" and "emotion" are often used interchangeably. However, they are distinct terms: "Feelings" relate to bodily sensations of light, sound, pain, balance, hunger, thirst, etc. generated by exposure to stimuli. Sensory perceptions are also accompanied by the release of neurotransmitters (NTs), attendant with body reactions and corresponding psychic states (Table 2). "Feeling" are pure perceptions that do not require memory, but they are remembered. Consequently, "emotions" can be considered as recalled "feelings".

Itskov et al hypothesized that the brain uses neural population activity to estimate elapsed time between events; the pattern of electrodynamic neural activity reflects context-dependent timing information. Buzaki, Eichenbaum, Moser and others described "time" and "place" cells, neurons that responded to temporal sequences as well as to space locations (Haftin et al, 2005; Buzsáki \& Moser, 2013 ; Silva et al, 2016).

There is no anatomic "sense of time", like for sight, sound, taste, etc. The hippocampus is central to the processing of incoming sensation. But missing is a satisfactory model for how the temporal order of emotive states is stored in the hippocampus (Yartsev \& Ulanovsky, 2013; Gonzalez, 2019).

The passage of time is intertwined with affective states. We rephrase our question:

By what physiologic process can the temporal sequence of emotive events be tied to molecular entities such as NTs)?

Time scale of Tripartite ordering of relative time:

The time scale for the interaction (binding or release) of elemental cations with the $\mathrm{nECM}$ is in the range of nanosecond $\left(10^{-7}\right.$ to $\left.10^{-9} \mathrm{~s}\right)$, appropriate for the dynamics of elemental diffusion and protein conformational changes. Thus, the many units of cognitive information encoded as [nECM/cation] arrays can feed into the synaptic and other neuronal signaling pathways (see Table 4).

Table 4: Kinetics of Neural processes.

\begin{tabular}{|c|c|}
\hline Process ${ }^{* \S}$ & Time scale \\
\hline Molecular conformation transition & $10^{-7} \mathrm{sec}$ \\
\hline Protein chain synthesis & $\begin{array}{l}10^{-1} \text { sec per amino } \\
\text { acid }\end{array}$ \\
\hline Neural firing rate & $10^{-2} \mathrm{sec}$ \\
\hline Neural firing rate & $300-400 / \mathrm{sec}$ \\
\hline Neuro-electric impulse ${ }^{\S}$ & $1-100 \mathrm{~m} / \mathrm{sec}$ \\
\hline Neural GPCR receptor diffusion & $10^{-1}$ to $10^{-3} \mathrm{um}_{2} \mathrm{sec}^{-1}$ \\
\hline RNA elongation & $10^{-2}$ sec per base \\
\hline DNA elongation & $10^{-3}$ sec per base \\
\hline $\mathrm{Ca}+2$ diffusion in $\mathrm{nECM}$ & $2.3 \times 10^{-6} \mathrm{~cm}^{2} / \mathrm{s}$ \\
\hline Protein turnover (replacement) & 3 months \\
\hline $\begin{array}{l}\text { Mosaic (sensor) diffusion over } \\
\text { neural surface }\end{array}$ & $10^{-1}$ to $10^{-3} \mathrm{um}^{2} / \mathrm{sec}$ \\
\hline
\end{tabular}

$\S$ Movie 24-30 frames/sec

*Ionic computer memory chip encoding 10-7 sec/bit

\section{Neural Signaling}

Synaptic Ever since the discovery of synapses by Cajal, these regions of the neural surface have been viewed as the channels whereby neurons communicate with one another. Synaptic contacts have also been considered as permitting electrodynamic potentials as a code for neural memory (Cajal, 1911; Cuentas-Condori \& Miller, 2020; Bailey \& Kandel, 2008). Notwithstanding, numerous doubts have been raised regarding the adequacy of synaptic signaling as a process that underlies neural memory (Arshavsky, 2006; Gallistel \& King 2009; Trettenbrein, 2016; Langille \& Brown, 2018).

\section{Ephaptic signaling}

We point out that the neurons also employ a non-synaptic, "ephaptic" mode of signaling (Jefferys, 1995; Bokil et al, 2001; Anastassiou, 2011). That is, neurons can eject vesicles containing NTs into the nECM to write emotive memory, (Marx \& Gilon, 2019) from all is surface. To read, the whole of the neural surface functions as a sensorium atuned to the exterior environment, namely the extracellular matrix (nECM) which functions as a "library" wherein the neural memory is J Psychol Neurosci; $2021 \quad$ www.unisciencepub.com Volume 3 Issue 2 
encoded and stored (i.e. see cuinfo, tripartite mechanism). The sensors (i.e. GPCR, AcholR) diffusing along the neural surface can detect and recognize the memory units (cuinfo) distributed within the nECM.

Assuming that such a tripartite mechanism is operative, we propose two processes that could achieve a perception of relative time as an exercise in memory.

cTime Mechanism \#1: Chemodynamic reading of relative time.

A series of cuinfo formed parallel to the neural membrane, can be detected by chemodynamic (allosteric) interactions between the sensors and the nECM (ref). Relative time is determined by the lateral placement of cuinfo along the neural surface. As they diffuse along the cell surface, the neural sensors (such as GPCR mosaics) are exposed to the extracellular cuinfo and perform as "reading heads". The "before" and "after" sequence is established in memory by the lateral reading order, as schematically represented in Figure 6.

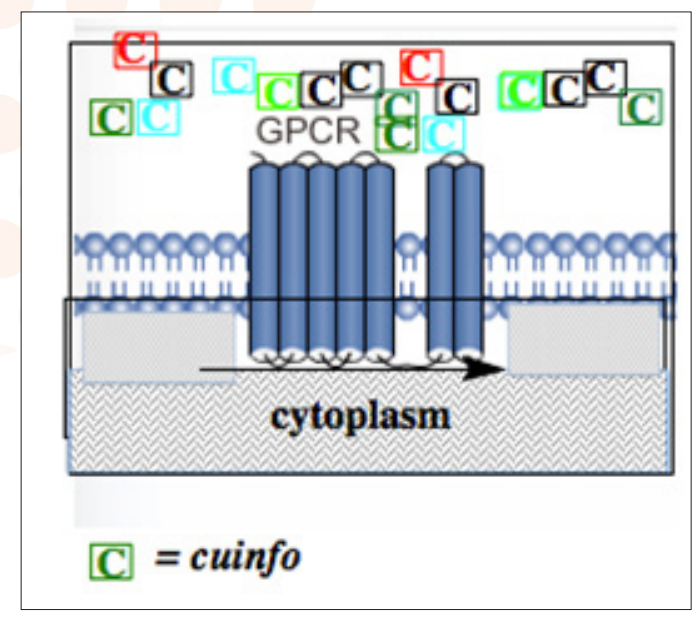

Figure 6: A GPCR sensor within the neural membrane "reading" the cuinfo around the neuron as it diffuses laterally $(\rightarrow)$ along the neural membrane. The different colors for the cuinfo ( $\mathbf{C})$ represent different emotive "flavors", accomplished by the incorporation of different NTs. Here, relative time is read by sensing the sequential lateral (left to right) placement of the cuinfo.

The panoply of neural sensors (i.e. GPCRs, K-channels and Acetylcholine receptors (ACholR) (Neumann, 2000) traversing the neural membrane perform as chemodynamic "readers" of the individual cuinfo. (Marx \& Gilon c, 2020). This suggests that there is a directional order in the formation of cuinfo and in the migration of the sensor mosaics to distinguish the "before" from the "after". The integration and consolidation of pixilated units from individual neurons, are performed by the neural net. In effect, this chemodynamic process provides a concept of how the memory of "relative time" could operate in neural creatures.

cTime Mechanism \#2. Electrodynamic reading of relative time.

An alternate option posits that the cuinfo are formed in vertical order, the latest unit being closest to the neural membrane; the older, more distant, further displaced vertically from the neural surface. Recall that the neuron (and its helper glia cells) continuously synthesize new nECM. Thus. Layers of nECM grow out and away from the neural surface. As soon as a cuinfo is formed, it is vertically displaced away from the neural surface by the biosynthesis of new "blank" nECM addresses, analgous to the growth of a coral reef.

One can hypothesize that reading vertically stacked cuinfo requires a field-sensing capability of the neurons analogous to the bioelectric field-sensing capabilities of electric fish and eels, which are capable bioelectric sensing of prey through many centimeter water (41-49) (Figure 6). Neurons generate a spatially and time-varying field of extracellular voltage (Ve) (Goldwyn \& Rinzel, 2016). There are numerous references to the ability of neurons to generate ephaptic fields (Neumann, 2000; Mankiewicz, 2010; ; Yao et al, 2009,2011; Agnati, et. al. 2014). The evolution of electric organs has evolved six times in fishes to produce electric fields for communication, navigation, predation, or signaling, leveraging similar transcription factors and developmental and cellular pathways (Gallant et al, 2014). Consider that neurons exert electrodynamic fields (Figure 8A), like electric fish (Heiligenberg, 1994) (Figure 7B), through many layers of nECM to detect near and distant cuinfo (Figure 7C).

Thus, there is ample precedent for considering that neurons employ electric fields to sense their distant surroundings. The neural surface membrane is covered with sensing mosaics, suggestive of a "neuro-theremin" effect. Neurons are like highly extended radio antannae which can send out and detect distal chemical fields. Think of radar, radio telescopes, remote detection technologies, RFID tags and theremin musical instruments (Martin et al, 2012) 


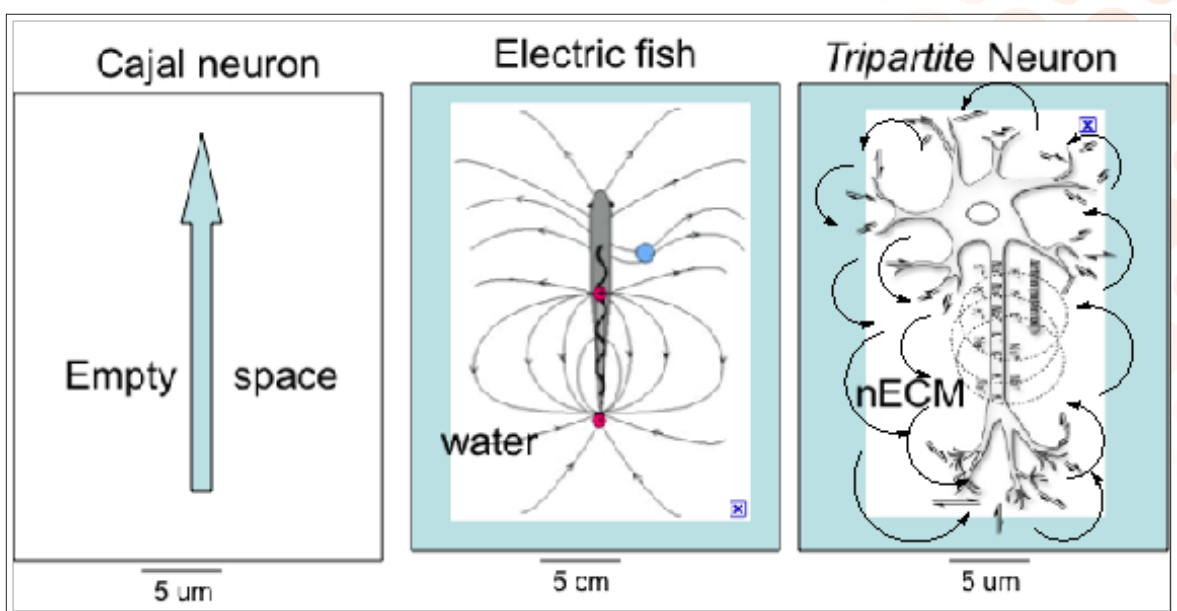

Figure 7: A. Cajal's schematic of a unidirectional signaling neuron (surrounding nECM not indicated) (i.e. "naked neuron").

This model is not physiologically realistic.

B. Electric field lines around an electric fish in water (Heiligenberg, 1994)).

C. Tripartite neuron with electric fields emanating into the nECM from various parts of its extended shape. The decoded "readings" are signaled to the neural net, and from there to the body, by synaptic pathways (adapted from: Mankiewicz R. 2010).

A horizontal or vertical layering of stacks of cuinfo adjacent to the neural surface (Figure $8 \mathrm{~A}, \mathrm{~B}$ ) suggests a possible means whereby temporal order (i.e. relative time) can be embodied in a neural system. The horizontal mode is based on a direct reading with GPCR or other sensors on the neural surface (Figure 8A). For the vertical mode, the neuron exerts an electric field by which it can sense distant regions of its environment, notably the vertically displaced cuinfo in the ever-expanding layers of the nECM (Figure $8 \mathrm{~B}$ ).

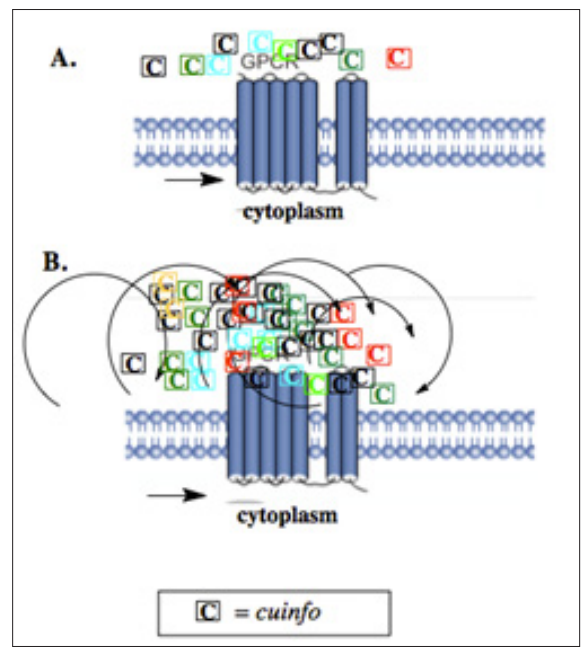

Figure 8: Two options for ordering the temporal sequence of neural memory.

A. Lateral ordering of monolayer of cuinfo parallel to the neural surface. Temporal order (relative time) is established via chemodynamic (allosteric) interactions of lateral sets of cuinfo with the moving receptor.

B. Vertical ordering of multilayered cuinfo extending from the neural surface, detected by a neuro-electric field. Temporal order (relative time) is established by the distance of the cuinfo from the neural surface.

Note that the variously colored cuinfo are tagged with molecular signifiers of emotions, namely NTs.
The tripartite mechanism provides a biochemical description of a possible process. It invokes physiologically relevant materials available to the neuron. It presents the neurotransmitters (NTs) as the elicitors and encoders of emotive states. Without memory, there are no emotions and visa versa...without emotive qualities, memory fades. Emotions and memory are inherently linked properties of active mentality.

\section{Complexity}

Saccharide-metal complex structures are complicated. The saccharide units can present a large number of monomeric saccharide units with metal-chelating groups (i.e. $\mathrm{OH}, \mathrm{COOH}$, $\mathrm{NH}_{2}, \mathrm{SO}_{3}^{-}, \mathrm{PO}_{3}^{-}$, etc.). As polymeric chondroitins, heparans and hyaluronates comprising combinatorial options of metal binding structures. For example, Figure 9 shows only a few of the types of structures capable of being achieved with different metal cations (Bandwar \& Rao,1996, 1997; Tachi et al, 2018; Rao et al, 2000; Kang et al, 2020 ; to cite only a few).

Consider that there are at least 10 physiologically relevant metals and more than 80 NTs. Along with the conformational flexibility of the nECM, they present near infinite combinatorial options for encoding cognitive information. 


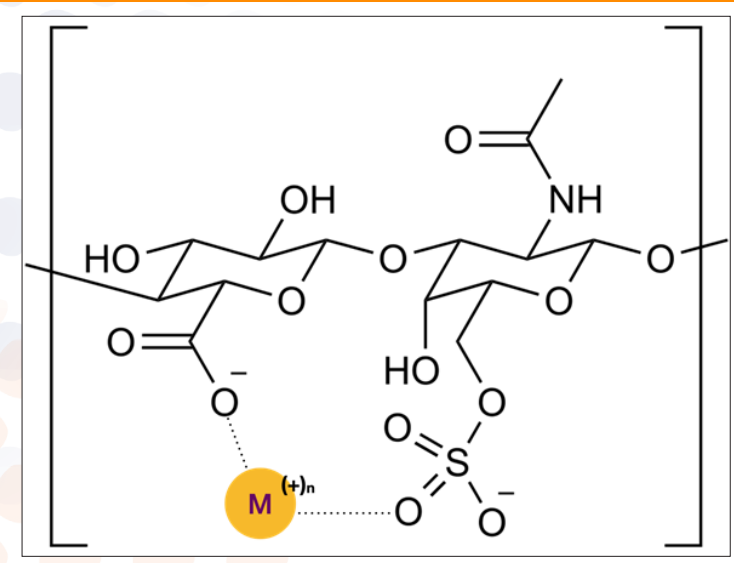

Figure 9: Schematic structure of chondroitin sulfate-metal complex. 2-D representations of disaccharide-metal complexes show the wide range of structural options.

The metal centers of such complexes also serve to anchor electron-rich NTs, to form ternary complexes with emotive qualities.(Adapted from: Allscher T., Klüfers P., Mayer P., Carbohydrate-Metal Complexes: Structural Chemistry of Stable Solution Species, In: Glycoscience. Fraser-Reid B, Tatsuta K, Thiem J (eds) Chapter-DOI 10-1007/978-3-540-30429-6_24: (C) SpringerVerlag Berlin Heidelberg 2008)

We are far from correlating exact molecular features of metalcomplexed nECM to a mental state associated with neural memory. Effectively, the nECM presents a limitless canvas for encoding cognitive-information tinged with emotive import as cuinfo. At this time, we are satisfied to have identified this class of molecular entities as relating to a mental state achieved by a neural net.

As to the connection of cTime to memory, we look to the tripartite mechanism of neural memory. It has the extraordinary virtue of introducing an aperture of molecular harmony to our comprehension of cTime. Based on a tripartite mechanism for the encoding of neural memory with cuinfo, the above schematics (Figure 9 A, B) suggest how the structured layering of cuinfo around individual neurons serves to establish a temporal hierarchy of memory with emotive "coloring"., as presented schematically in Figure 9.

\section{Conclusion}

The "trinity" of Past, Present and Future presents a conceptual challenge. We do not have an anatomic sense of time in the same way that we can sense color, smell, touch or heat, each of which can be mechanistically described.

Q: To what biological process does the sense of time relate?

Time is linked to memory. A mechanism of neural memory could provide an overarching rationale for the ability of neural nets to recall the temporal sequence of past stimuli. For nonverbal animals, absolute "time" does not exist. As it does not have materiality, "time" does not literally exist anyway. Rather, "Time" is a verbal construct, of no consequence except to homo sapiens, who invented the word and the concept (i.e. Global UTC). For neural creatures, "relative time" is an operative phenomenon tied to emotions. The timing of emotive events, the kinetics and speed of now and later are what count for in the life and memory of all animals. Though "place cells" and "time cells" in the hippocampus are involved in the recognition of place and "Time" (Hafting et al, 2005; Yartsev \& Ulanovsky, 2013), we still desire a molecular accounting for the temporal ordering and persistence of emotive memory.

Minkowski's treatment of Space-Time (Smart, 1964), invoked 2 points:

- Does it contradict experience?

- Is it advantageous for describing phenomena?

We adapt these points for our purpose, as follows: The tripartite mechanism of neural memory invokes 2 neuro-biological components of which Cajal was not aware:

- The various trace metals which are critical to the mentation process

- The nECM mesh surrounding the neurons, which Cajal's staining method did not reveal (both reviewed in Marx \& Gilon, 2012-2020).

Granted that the emotive dimensions of mentality are based on physiologic reactions entangled with psychic states, how is this enabled? We point out that chemicals (neurotransmitters (NTs) and drugs) are the only molecular entities capable of eliciting such responses from animate beings.

One should be courageous and admit that the metrics of physics are not adequate to describe mental states; that "consciousness" occupies a novel role in the laws of Nature. A new paradigm is needed to account for the transformations of metabolic energies into new mental dimensions manifest as subjective experience entangled with timed reactions.

This thesis is admittedly speculative but is presented to stimulate further discussion. Also, it may suggest novel tests of the tripartite mechanism. For example, it would be of interest to determine the molecular structures of tetra saccharidemetal-NT complexes by X-ray diffraction, to lend credibility to the molecular approach to comprehending neural memory. Another track would be to continue working with coated impedimetric electrodes, whereby we previously demonstrated that complexation of metals with oxytocin and tetrasachharide analogues of hyaluronic acid could modulate electrode activity (Tadi et al, 2017; Alshansky et al, 2019; Marx \& Gilon, 2021).

\section{The horizon is vast \\ The voyage is long \\ Time awaits.}

\section{Acknowledgement}

(By GM). With gratitude to my wife the artist Georgette Batlle (1940-2009), and my children Danae and Jonathan, who always looked kindly on me during the years of my travails relating to trace metals, blood coagulation, art and "thought". Thanks to friends, the late Bill Needle (Eastchester, N.Y.) and Lilly Rivlin (New York, N.Y.) for their early encouragement and financial support in the period 1980-1984. Lilly recently sent me this appropriate message: "My film is screening in many places and my time is limited in more ways than one". 
Thanks to Ahouva Leopold (Jerusalem, Paris) who engages me in discussions of objective, subjective, spiritual and virtual time, and for introductions to people and places. I appreciate Professor Jean- Paul Doguet's (Paris) interest and suggestions regarding the relevance of the philosophers Husserl and Bergson.

\section{Conflict of Interest}

GM is a founder of MX Biotech Ltd., with the commercial goal to develop new "memory materials" and devices.

CG is an emeritus professor at the Institute of Chemistry, The Hebrew University of Jerusalem. He is active in developing technologies for the conversion of peptides and active regions of proteins into orally available drugs.

Notwithstanding, the ideas forwarded here are scientifically genuine and presented in good faith, without commercial clouding of the concepts expressed therein.

\section{References}

1. Agnati, L.F., et. al (2014) Information handling by the brain: Proposal of a new "paradigm" involving the roamer type of volume transmission and the tunneling nanotube type of wiring transmission. J. Neural Transm. (Vienna) 121(12): 1431-1449. doi: 10.1007/s00702-014-1240-0.

2. Amtul Z., Rahman A. (2016) Neural Plasticity and Memory: Is Memory Encoded in Hydrogen Bonding Patterns? The Neuroscientist 22: 9-18.

3. Arbib, (1987) Brains, Machines and Mathematics. Springer Verlag, Berlin.

4. Bergson H (1908) Matter and Memory, Dover Publications, New York Translation from 1912 Edition. Allen \& Co, London.

5. Block N, Hannagan O, Guzeldere G. (Eds). (1997) The Nature of Consciousness. Philosophical Debates. MIT Press, Cambridge, MA.

6. Brand S (1999) The Clock of the Long Now. Time and Responsibility. Phoenix Paperbacks, UK.

7. Brann E. (1999) What Then, Is Time? Rowan \& Littlefield, MD.

8. Brown JW (1990) Psychology of time awareness. Brain Cogn. 14: 144-164.

9. Bueti, D. (2011) The sensory representation of time. Front Integr Neurosci 5: 34. doi: 10.3389/fnint.2011.00034

10. Burdick A. (2017) Why Time Flies. Simon \& Schuster, New York.

11. Buzsáki G, Moser EI (2013) Memory, navigation and theta rhythm in the hippocampal-entorhinal system. Nat Neurosci. 16: 130-138. DOI: 10.1038/nn.3304 [PubMed: 23354386

12. Buzsaki G., Llinas R. (2017) Space and time in the brain. Science 358: 482-485.

13. Cajal R.Y (1911) Histology of the Nervous System of Man and Vertebrates, Oxford University Press,1995 ISBN: 9780195074017

14. Cuentas-Condori A., Miller, DM 3rd (2020) Synaptic remodeling, lessons from C.elegans. J. Neurogenetics
34:307-322, DOI:10.1080/01677063.2020.1802725

15. Cho, A. (2019) Tests measure progress of quantum computers. Science 364: 1218-1219.

16. Coleman GA, Davín AA, Mahendrarajah TA, Szánthó LL, Spang A, Hugenholtz P, Szöllősi GJ, Williams TA. (2021) A rooted phylogeny resolves early bacterial evolution. Science 372: 588.

17. De Warren N (2016) Augustine and Husserl on Time and Memory. Quaestiones Disputatae 7: 7-46.

18. Di Ventra M., Pershin Y.Y. (2011).Memory materials: A unifying description. Materialstoday 14: 584-591. Doi: 10.1016/S1369-7021(11)70299-1

19. Eagleman D.M., Tse P.U., Buonomano D., Janssen P., Nobre C.A., Holcombe A.O. (2005) Time and the brain: How subjective time relates to neural time. J. Neuroscience 25: $10369-10371$.

20. Eichenbaum H. (2013) Memory on time. Trends Cogn. Sci. 17: 81-88. doi: 10.1016/j.tics.2012.12.007.

21. Elman JL (1990) Finding structure in time. Cognitive Science 14: 179-211.

22. Faivre N, Koch C. (2014) Temporal structure coding with and without awareness. Cognition 131: 404-414.

23. Fischer R. (Ed.) (1967) Interdisciplinary Perspectives of Time. New York Acad. Sci 138: 367-915.

- Fischer R. The biological fabric of Time. pp 440-488.

- Sollberger A. Biologic measurements in Time with particular reference to synchronization mechanisms.

- $\quad$ Oswald I. Slow neurophysiological Time. pp 616-622.

- Schaltenbrand G. Consciousness and time. pp 632-645.

24. Fodor J.A. (1975) The Language of Thought. TY Crowell Co. Inc. USA.

25. Fodor J.A. (1983)The Modularity of Mind. MIT Press, Cambridge, MA.

26. Franklin S. (1995) Artificial Minds. MIT Press, Cambridge, MA.

27. Fraser JT. (1987) Time: The Familiar Stranger. Tempus Books, Washington DC.

28. Gallistel CR, Gibbon J. (2000) Time, rate, and conditioning. Psychol. Rev. 107: 289-344.

29. Gerstner, J. R. (2012) On the evolution of memory: a time for (engrams and) clocks. Front. Mol. Neurosci. 5 : 1-9. 23. doi: 10.3389/fnmol.2012.00023.

30. Gibbon J, Church RM, Meck WH (1984) Scalar timing in memory. Ann. N.Y. Acad Sci. 423: 52-77.

31. Gonzalez WG, Zhang H., Harutyunyan A., Lois C. (2019) Persistence of neuronal representations through time and damage in the hippocampus, Science 365: 821-825.

32. Gupta D.S., Merchant, H. (2017) Editorial: Understanding the role of the time dimension in the brain information processing. Front. Psychol.doi: 10.3389/fpsyg.2017.00240

33. Hafting T, Fyhn M, Molden S, Moser M-B, Moser EI. (2005) Microstructure of a spatial map in the entorhinal cortex. Nature. 436: 801-806. DOI: 10.1038/nature03721 [PubMed: 15965463

34. Heiligenberg W. (1994) The coding and processing of temporal information in the electric fish. In: Buzsaki G, Llinas R., Singer W., Berthoz A., Christen Y. (Eds). Temporal Coding in the Brain. Springer Verlag, Berlin. 
35. Howard N. (2012) Energy paradox of the brain. The Brain Sciences Journal 1: 46-61.

36. Joyce R. (1997) Cartesian memory, J History Philos. 35(3): 375-394.

37. Katchalsky A., Neumann E. (1972) Hysteresis and molecular memory record, Int. J. Neuroscience 3(4): 175182. DOI: $10.3109 / 00207457209147020$

38. Kim J. (2000) Mind in a Physical World. An Essay on the Mind-Body Problem and Mental Causation. MIT Press, MA

39. Landauer R. 1991; 1996.

- Information is physical.

- $\quad$ Physics Today; May, p 93-29.

- The physical nature of information

- $\quad$ Physics Letters A 217: 188-193

40. Lin M. (2005) Memory and personal identity in Spinoza, Canadian Journal of Philosophy 35: 243 - 268.

41. Maass W, et al. (2007) Computational aspects of feedback in neural circuits. PLoS Comput. Biol.

42. Maniadakis M., Trahanias P. (2016) When and How-Long: A Unified Approach for Time Perception. Front. Psychol., | https://doi.org/10.3389/fpsyg.2016.00466

43. Martín C.B., Martínez J.S., Ricchiuti A., González H.E., Franco C.H. (2012) Study of the interference affecting the performance of the Theremin. Int. J. Antennas and Propagation, Article ID 348151. doi.org/10.1155/2012/348151

44. Marx G., Gilon C. (2012) The molecular basis of memory. ACS Chemical Neuroscience 3: 633-642.

45. Marx G., Gilon C. (2013) The molecular basis of memory. MBM Pt 2: The chemistry of the tripartite mechanism. ACS Chem Neurosci 4: 983-993.

46. Marx G., Gilon C. (2014). The molecular basis of memory. MBM Pt 3: Tagging with neurotransmitters (NT). Frontiers in Neurobiology 6: 1-8.

47. Marx G, Gilon C. (2020) The Molecular Basis of Neural Memory. Part 11. Chem-electric Write /Read Processes. J. Neurosurg. Imaging Techniques 6: 283-301.

48. Marx G, Gilon C. (2020) Tripartite mechanism of neural memory: Proof-of-concept with neuromimetic impedance electrodes. Biomedical Research and Clinical Review 1(3): 021, DOI: 10.31579/2692-9406/021

49. McCulloch WS, Pitts W. (1943) A logical calculus of the ideas immanent in nervous activity. Bulletin of Mathematical Biophysics 7: 115-133.

50. Reprinted in: McCulloch Embodiments of Mind, Cambridge 1988 ; MIT Press

51. Muller R.A. (2016) Now: The Physics of Time. Norton \& Co., New York. Pauling L. 1960. The Nature of the Chemical Bond. $3^{\text {rd }}$ Ed. Cornell University Press, Ithaca, NY

52. Pockett S. (2014) Problems with theories that equate consciousness with information or information processing. Front. Syst. Neurosci. 8: 1-3. doi: 10.3389/fnsys.2014.00225

53. Ray S., Valekunja U.K, Stangherlin A, Howell S.A., Snijders A.P., Damodaran G., Reddy A.B. (2020) Circadian rhythms in the absence of the clock gene Bmall. Science
367(6479): 800-806. doi: 10.1126/science.aaw7365.

54. Rosenblum B, Kuttner F (2011) Quantum Enigma: Physics Encounters Consciousness. 2 ${ }^{\text {nd }}$ Ed. Oxford University Press, Cambridge, UK. Schrodinger E. 1944.

55. Mind and Matter. In: What is Life? Cambridge University Press, UK

56. Shannon. CE. (1948) A mathematical theory of communication, The Bell System Technical Journal 27: 379-423, 623-656.

57. Singer BR. (1976) Hooke on memory, association and time perception (1820). Notes Rec. R. Soc. Lond. 31: 115 - 131. (doi:10.1098/rsnr.1976.0003)

58. Singer W. (1994) Time as coding space in neocortical processing: A hypothesis. In: Buzsaki G., Llinas R., Singer W., Berthoz A, Christen Y. (Eds).Temporal Coding in the Brain. Springer Verlag, Berlin.

59. Smart J.J. (Ed).(1979) Problems of Space and Time. [Essays by St. Augustine, Descartes, Newton, Locke, Kant, Mach, Russell, Eddington, Einstein, Minkowski and others]. MacMillan Publishing Co. New York.

60. Street S. (2016) Neurobiology as information physics. Front. System Neurobiol. 10: 90 Doi: 10.3389. fnsys 2016 00090

61. Taub (Ed). (1950) Von Neumann Collected works, The Natural and Logical Theory of Automata. Volume V Design of computers, theory of automata and numerical analysis.

62. Tononi G. (2004) An information integration theory of consciousness. BMC Neuroscience, 5: 42 doi:10.1186/1471-2202-5-42

63. Tononi G. (2008) Consciousness as integrated information: A provisional manifesto. Biol Bull. 15: 216-242.

64. Torday JS, Miller Jr. WB. (2016) On the evolution of the mammalian brain. Front. Syst. Neurosci. 10: doi: $10.3389 /$ fnsys. 2016

65. Tulving E. (2002) Episodic memory: From mind to brain. Ann. Rev. Psychol. 53: 1-25.

66. Turing AM. (1950) Computing machinery and intelligence. Mind (New Series) 59: 23: 433-460. Pergamon Press, New York.

67. Wittmann M. (2013) The inner sense of time: How the brain creates a representation of duration. Nature Reviews Neuroscience 14: 217-223.

68. Wittmann M. (2016) Felt Time. The Psychology of How We Perceive Time. MIT Press, Cambridge. MA.

69. Wray GA. (2015) Molecular clocks and the early evolution of metazoan nervous systems. Philos Trans $R$ Soc Lond B Biol Sci; 370. doi: 10.1098/rstb.2015.0046.doi: 10.1098/ rstb.2015.0046.

70. Yartsev MM, Ulanovsky N. (2013) Representation of three-dimensional space in the hippocampus of flying bats. Science 340: 367-372. doi: 10.1126/science.1235338.

Copyright: (C2021 Gerard Marx. This is an open-access article distributed under the terms of the Creative Commons Attribution License, which permits unrestricted use, distribution, and reproduction in anymedium, provided the original author and source are credited. 